\title{
Predictive System for Medical Emergency and Admissions Control
}

\author{
G. Subhashini, V. Neelambary
}

\begin{abstract}
An emergency department (ED), provides specialized facility in emergency medicine they are the life saving one for many people in emergency cases. Some main issues in ED's are overcrowding, quality of care for the patients etc., here overcrowding is still a big issue in most of the hospitals. This paper implements some advanced methods to improve patient flow and prevent overcrowding. The ED overcrowding can be reduced by predicting the future of patient's admission using Machine Learning and making the resources available beforehand. This technique help us to learn, analyze and predicting the future results. In this work we implement Predictive Gradient Boosted Machines (PGBM) which produces a prediction model in the form decision trees. The data set which we use in this paper has several factors like hospital admissions, including site of the hospital, patient's age, mode of arrival, previous admission in past month and past year. Decision Trees creates a training model which predicts the data by understanding decision rules that are observed from training data. The accuracy of the system is achieved by implementing Deep Neural Network (DNN) which uses efficient mathematical model to process data in complex ways.
\end{abstract}

Keywords: Emergency department, Machine Learning, Predictive Gradient Boosted Machines, Decision Trees, Deep Neural Network.

\section{INTRODUCTION}

Artificial intelligence and Machine learning are playing a vital role in the hospitals. Hospitals faces many problems one of the problem which we concentrate here is the overcrowding in ED. Overcrowding in ED is one of the biggest problem in many hospitals across the world. It has serious issues in admission of patient in terms of more waiting time, ambulance diversion to some other hospital due to which there can increase mortality of the patients. There are some reasons of overcrowding in hospitals they are less doctors or attendees during the night hours, lack of alternate treatment, and lack of inpatients beds, ED staffing shortages and closure of other local ED departments. The most important of these causes is manage the patient flow so that patient can be admitted and also the capacity of the inpatients also to be understand.

One of the mechanism that helps to reduce ED crowding and improve the patient flow by using data mining. Using this technique we can identify the patients who are in high

Revised Manuscript Received on November 22, 2019.

* Correspondence Author

Ms.G.Subhashini, Assistant Professor, Department of Information Technology, St.Joseph's Institute of Technology, Affliated to Anna University, Chennai, India. Email: gopal.subha191@gmail.com

Ms.V.Neelambary, Assistant Professor, Department of Information Technology, St.Joseph's Institute of Technology, Affliated to Anna University, Chennai, India. Email: neelambaryvasu@gmail.com

risk. Data mining techniques, this technique includes examining and analyzing data to extract useful information and knowledge on which decisions can be taken. It involves identifying and describing patterns in the data and predicts the result based on the past patterns. But by using data mining the prediction was less accurate. In order to overcome this Machine learning was used where the system get trained and allows accurate prediction. To improve accuracy still more Deep Neural Network is used. Patients who approach the ED undergo through several phases between the arrival and discharge time depending on decisions made at preceding stages. The patients of ED can arrive to the hospital either via through the reception area or in an ambulance. The patient's details are stored on the hospitals administrative database, the patients information are collected before admitting the patient, or the can be made waited in the waiting area. The waiting time cannot exceed fifteen to twenty minutes if exceeding a nurse will be assisting the patient. The Manchester Triage scale are used by many hospitals of the Northern Ireland , in this method patients are admitted by prioritizing patients mainly on the severity of their condition, and to identify patients emergency. Triage system is one of the best method used in many of the hospitals and reduce the death rates to a great extend.

Triage system is one of the most reliable method in predicting the admission in the hospital's emergency department but this system is reliable in the less crowd but not when it is highly scalable. In this triage system once the patient reach the hospital his emergency is analyzed based on the analysis the patients are served, some patients are send to the waiting room before he is assisted by a clinician. The clinician will make some recommendation or first aid to the patients. If there is need to admit the patient, the emergency department sends a request for the bed and the patients waits until the bed is available. Overcrowding can be resulted in case of bottlenecks and excess demand. Patients are completely monitored in the ED and each and every phases of the patient's data are recorded routinely in the system. This system is further more extended to control the admissions by using Machine Learning techniques. This study focuses on the use of Deep Neural Network algorithms to develop models by predicting hospital admissions from the emergency department. This system meets the three basic requirements, i.e., resource allocations, avoid bottleneck, and to improve the ED admissions efficiently. The efficiency of the system is further improved by improvising the system through Deep Neural Network and achieving the efficient ED admissions. 


\section{Predictive System for Medical Emergency and Admissions Control}

\section{RELATED WORK}

Ron graham et al., proposed logistic regression to predict the admissions in ED, they identified several factors related to hospital admissions, including hospital site, age, arrival mode, triage category, care group, previous admission in the past month, and previous admission in the past year. In this work they utilized three common machine learning algorithms in predicting patient admissions. The implementation of the system explains about the advanced resource allocation and planning and avoiding the bottlenecks. The efficiency of the method was improved by data learning algorithms. The drawback of this system is accuracy was moderate. [1]

J. Boyle et al. proposed a model of historical data analysis this data is depended on the past 5 years of historical data collected from two different hospitals. In these hospitals the emergency records are collected and analyzed patients past history and the details about the medicines and the treatment provided along with their medical condition. Based on all these factors the admissions are controlled. The accuracy that is measured are assessed using the mean average percentage error (MAPE) that compares the forecasted and recorded data. A threshold is recorded for the analyzed data .The historical method is highly efficient but the drawback was some patients were diverted to different hospitals due to insufficient beds. Proper bed management is needed in this system. The paper provides forecasting performance levels to guide similar studies.[2]

M. L. McCarthy et al Triage system is one of the most reliable method in predicting the admission in the hospital's emergency department but this system is reliable in the less crowd but not when it is highly scalable. In this triage system once the patient reach the hospital his emergency is analyzed based on the analysis the patients are served, some patients are send to the waiting room before he is assisted by a clinician. The clinician will make some recommendation or first aid to the patients. If there is need to admit the patient, the emergency department sends a request for the bed and the patients waits until the bed is available. Overcrowding can be resulted in case of bottlenecks and excess demand. The drawback in the system is overcrowding is not handled efficiently. [10]

F. R. Lucini et al., proposed a study, which makes use of text mining methods were used to process data from past ED patient records. This uses a SOAP framework, and predict future admission, care and discharges. The design of the system was first to preprocess the record of text and predict the admissions. The text are converted to binary representation and the term frequency is also recorded. The results are tested by various text testing techniques like unigrams, bigrams and trigrams. Here eight models are tested Decision Tree, Random Forest, Extremely Randomized Tree, AdaBoost, Logistic Regression, Multinomial Naïve Bayes, Support Vector Machine (Kernel linear) and $\mathrm{Nu}$-Support Vector Machine (Kernel linear).The method could be used to manage daily routines in EDs such as capacity planning and resource allocation. Text mining could provide valuable information and facilitate decision-making by inward bed management teams. [11]

\section{Proposed SySTeM}

The Proposed system increases the prediction of patient admission in the ED by reducing the bottleneck. The system has an additional classification called Deep Neural Network algorithm. This increases the accuracy of the new system. The Perceptron model is a feed forward neural network model capable of classification and regression. This perceptron is composed of fully connected layers of artificial neurons forming a connected graph, where neurons are modelled very loosely on biological neurons; and as a feed forward net, the output of each layer is given as the input of the next one. The training is done through the back propagation Algorithm this is an application of gradient descent that takes into account the contribution to the error by the neurons of the hidden layer. A data set is collected from a hospital database. The data set has the details about the patient current and previous history, based on this data the patients emergency is analyzed this results in the patients criticalness. This criticalness report is used as an input to provide treatment to the patient on his emergency. Adding the proposed system to the old system increases the predictions by reducing the over fitting.

a. Advantages:

- This system avoids the bottleneck situations and also helps in maintain the patient flow in the hospital.

- System has an additional classification and also helps in predicting resources allocation for patients.

- DNN used in this system increases the prediction accuracy of the system.

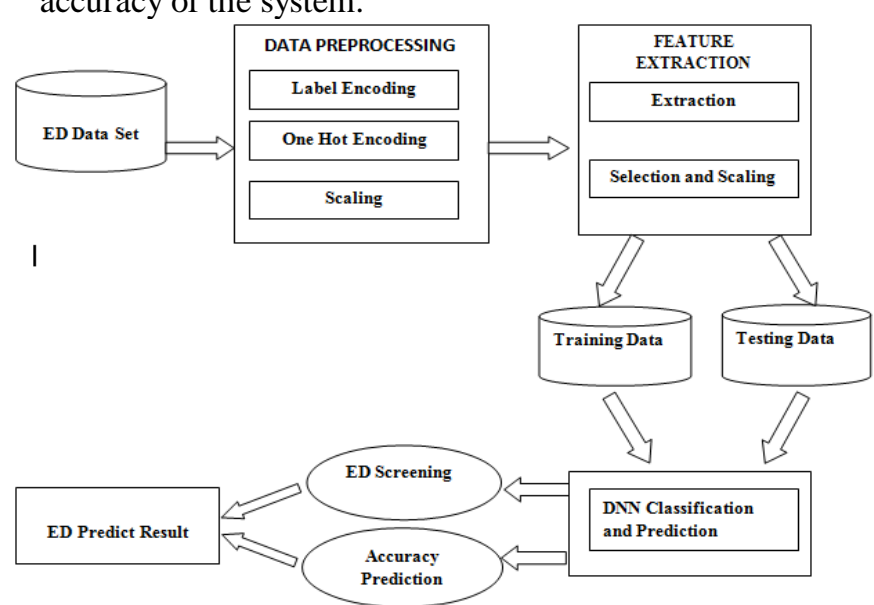

Fig 3.1 System Architecture diagram for Emergency Department

Fig 3.1 describes about the system architecture for emergency department. Initially the hospital data set is collected, then it is preprocessed using three method label encoding, One Hot encoding and scaling. This produces a preprocessed data where the unwanted irrelevant data are removed and a structured data is generated. Next the preprocessed data is send to the feature extraction phase.

The resultant data is split into training and testing data. These data are classified and predicted using DNN. The following modules are the implementation of the system. 


\section{A. Preprocessing}

The data has to Cleaned before loading into the Neural Classifier. Preprocessing involves three methods Label encoding, One hot encoding and Standard Scaler. Label Encoding are used to convert categorical data, or text data, into numbers, which our predictive models can better understand. Import the Label Encoder class from the sklearn library, fit and transform the data, and then replace the existing text data with the new encoded data. Label encoding generates the result in the form given below.

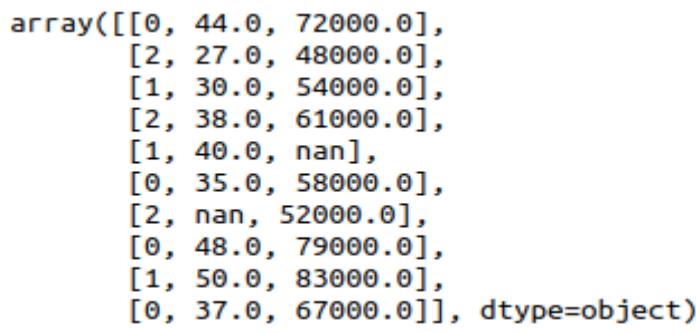

Fig: 4.2 Label encoding result

One hot encoding is a representation of categorical variables as binary vectors. Categorical variables are converted into a form that could be provided to Machine Learning algorithms to do a better job in prediction. StandardScaler is the graphical representation that assumes the data is normally distributed within each feature and will scale them such that the distribution is now around 0 with a standard deviation of
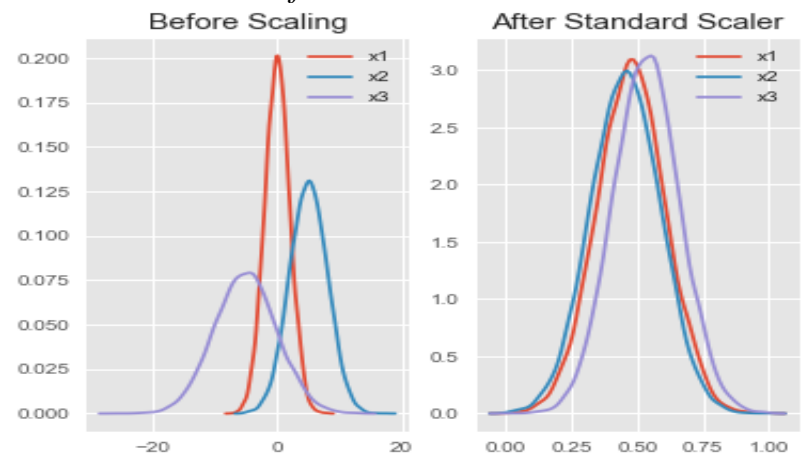

\section{B. Data visualization and descriptive statistics:}

The extracted features are usually plotted against the output to check its relation to the output .

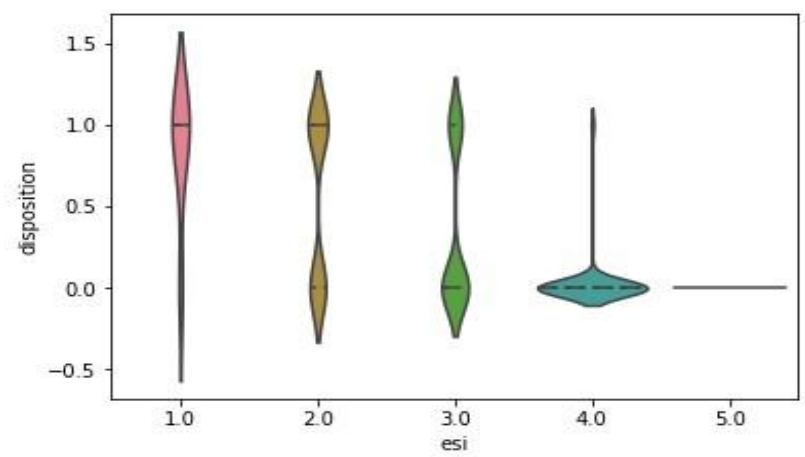

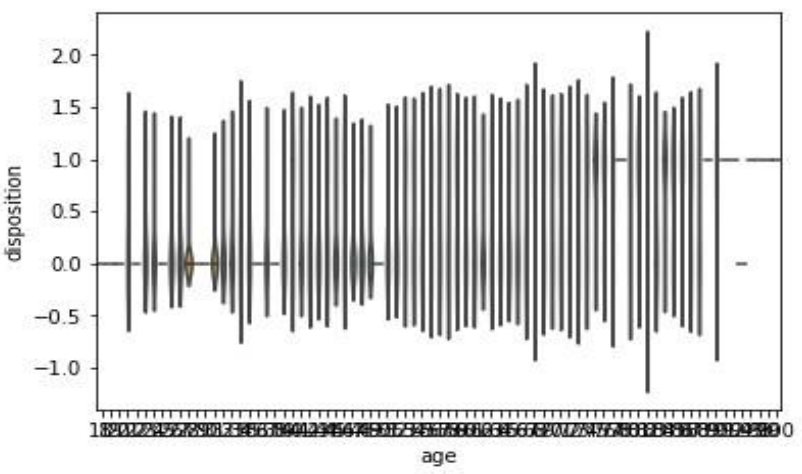

Fig 3.2 Disposition vs ESI and Age vs Disposition

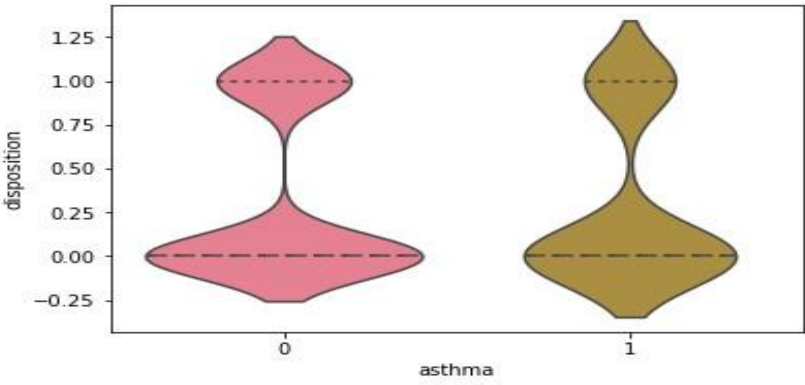

Fig 3.3 Asthma vs Disposition

\section{Data Splitting}

Data splitting is the process of splitting the data into training data and testing data. This splitting may result in overfit or underfit this affects the prediction. To overcome this problem we use DNN to improve the accuracy.

\section{Predictive Admissions}

This system uses Deep Neural Network (DNN) for performing predictions.DNN is one of the efficient technology that is used to process the data in complex ways. Back-propagation is $s$ a method to compute the partial derivatives of a function, which has the form as a function composition. When you solve an optimization problem using a gradient-based method (gradient descent is just one of them), you want to compute the function gradient at each iteration. In this technique Analytic differentiation is used that just compute the derivatives using the chain rule (basic calculus). In this system the overcrowding is reduced by admitting the patient by predicted data of past history. 


\section{Predictive System for Medical Emergency and Admissions Control}

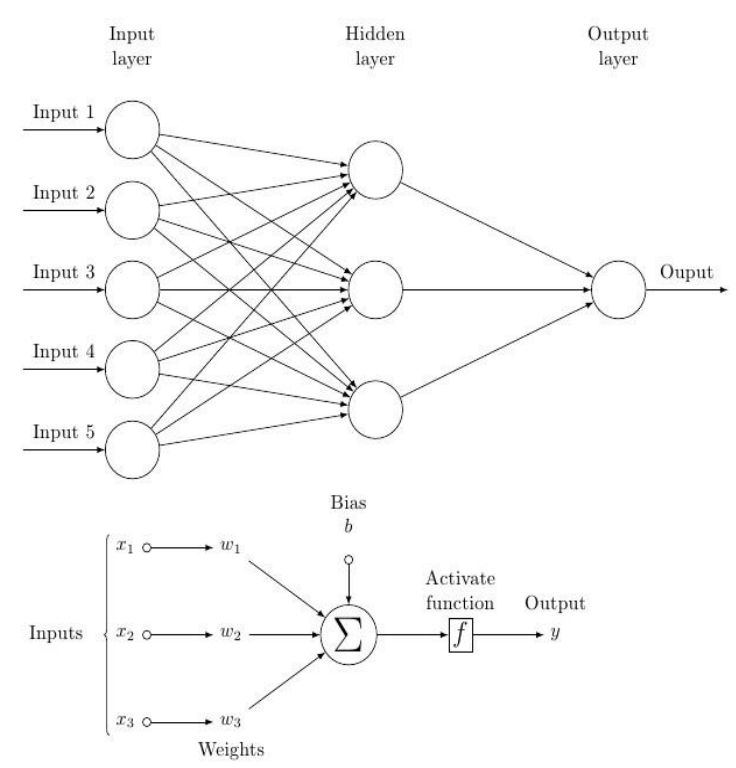

Fig3.4: Deep Neural Network

\section{E. Evaluation of model performance}

This is the final step where we are evaluating our model performance. We build Confusion matrix to check the accuracy of model. Confusion matrix is a two by two matrix that has four outcomes produced by a binary classifier. Various measures, such as error-rate, accuracy, specificity, sensitivity, and precision, can be obtained from the confusion matrix. The accuracy is calculated using the following formula. TP-True Positive, TN-True Negative, P-Positive and N-Negative

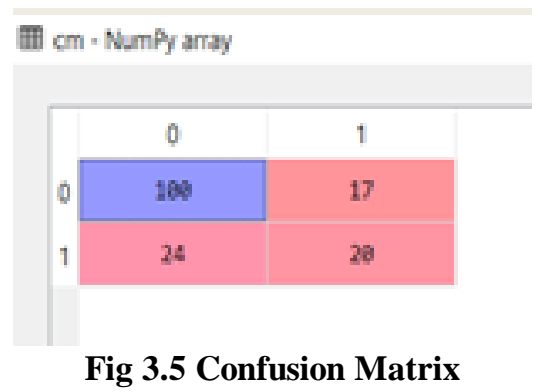

Accuracy $=(\mathrm{TP}+\mathrm{TN}) /(\mathrm{P}+\mathrm{N})$

Accuracy $=100+20 / 2000=0.7453416=74.5 \%$

\section{CONCLUSION}

Thus, the system helps in predicting the admission of patients and also determining the Resource allocation for the patients. The chaos is prevented by predicting the disposition of patients. Confusion matrix is used to check the accuracy of the model. Deep Neural Network is used to predict the disposition of the patients and K-Nearest Neighbors is used to predict the resource allocation for the patients. Tensorflow and theano are Machine learning libraries of Keras used at the background to train the dataset in sequential model of Deep Neural Network.

\section{REFERENCES}

1. Ron graham, raymond bond, michael quinn, and maurice mulvenna, "Using Data Mining to Predict Hospital Admissions From the Emergency Department" (2018), doi: 10.1109/ACCESS.2018.2808843.

2. J.Boyle et al., "Predicting emergency department admissions," Emerg. Med. J., vol. 29, pp. 358-365, May 2012, doi: 10.1136/emj.2010.103531.

3. Cameron, A. J. Ireland, G. A. McKay, A. Stark, and D. J. Lowe, 'Predicting admission at triage: Are nurses better than a simple objective score?''Emerg. Med. J., vol. 34, pp. 2-7, Feb. 2016, doi: 10.1136/emermed-2014-204455

4. M.-L. Connolly. (2015). NI Emergency Healthcare Enquiry Finds 'Degrading' Cases. Accessed: Oct. 14, 2015. [Online].

5. NHS England. (Jun. 2015). A\&E attendances and Emergency Admissions' June 2015 Monthly Report. [Online]. Available: DHSSPS. (2015) Northern Ireland Hospital Statistics: Emergency Care (2014/15), Northern Ireland.

6. N. Esfandiari, M. R. Babavalian, A. M. E. Moghadam, and V. K. Tabar, "Knowledge discovery in medicine: Current issue and future trend,"

7. B. Xie, "Development and validation of models to predict hospital admission for emergency department patients,'” Lifescience Global, vol. 2, no. 1 , pp. $55-66$.

8. J. Boyle et al., "Predicting emergency department admissions," Emerg. Med. J., vol.29, pp. 358-365, May 2012, doi: 10.1136/emj.2010.103531

9. Y. Sun, B. H. Heng, S. Y. Tay, and E. Seow, "Predicting hospital admissions at emergency department triage using routine administrative data," Acad. Emerg. Med., vol. 18, no. 8, pp. 844-850, 2011, doi: 10.1111/j.1553-

10. M. L. McCarthy et al., "Crowding delays treatment and lengthens emergency department length of stay, even among high-acuity patients,' Ann. Emerg Med., vol. 54, no. 4, pp. 492-503, 2009, doi 10.1016/j.annemergmed.

11. F. R. Lucini et al., "Text mining approach to predict hospital admissions using early medical records from the emergency department,' Int. J. Med. Inf., vol. 100, pp. 1-8, Apr. 2017, doi: 10.1016/j.ijmedinf.2017.01.001.

12. J.-A. Huang, R.-H. Weng, W.-C. Tsai, W.-H. Hu, and D.-Y. Yang, "Analysis of emergency department utilization by elderly patients under national health insurance,'” Kaohsiung J. Med. Sci., vol. 19, pp. 113-120, Mar. 2003, doi: 10.1016/S1607-551X(09)70458-9.

13. S. W. Kim, J. Y. Li, P. Hakendorf, D. J. O. Teubner, D. I. Ben-Tovim, and C. H. Thompson, "Predicting admission of patients by their presentation to the emergency department,'” Emerg. Med. Austral., vol. 26, no. 4, pp. 361-367, 2014, doi: 10.1111/1742-6723.12252

14. J. S. Peck, J. C. Benneyan, D. J. Nightingale, and S. A. Gaehde, "Predicting emergency department inpatient admissions to improve same-day patient flow," Acad. Emerg. Med., vol. 19, no. 9, pp. 1045-1054, 2012, doi: 10.1111/j.1553-2712.2012.01435.x.

15. Arel, D. C. Rose, and T. P. Karnowski, "Deep machine learning-A new Frontier in artificial intelligence research," IEEE Comput. Intell. Mag. vol. 5, no. 4, pp. 13-18, Nov. 2010, doi: 10.1109/MCI.2010.938364.

\section{AUTHORS PROFILE}

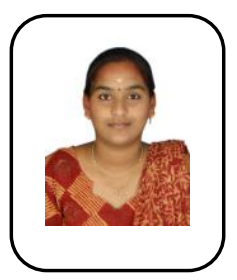

Ms. G. Subhashini , ME(CSE) Assistant Professor St.Joseph's Institute of Technology. Published papers in journals like IJRTE, IJREAT presented papers in various National and International Conferences. Area of Specialization is Machine Learning and Medical Imaging. One of the coordinator of Computer Society of India (CSI).

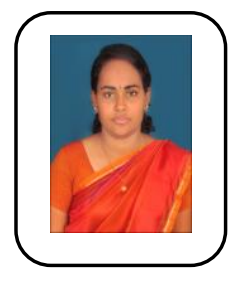

Ms. V. Neelambary, ME(SE) Assistant Professor St.Joseph's Institute of Technology. Published papers in journals like IJRTE, IJREAT presented papers in various National and International Conferences. Area of Specialization is Machine Learning and software Engineering. One of the coordinator of Computer Society of India (CSI) and Institution of Engineers (India) (IEI). 